\title{
Uniform Sampling of ECG Waveform of MIT-BIH Normal Sinus Rhythm Database at Desired Intervals
}

\author{
Jitu Sharma \\ BIET, Jhansi \\ Jhansi, UP, India \\ Shahanaz Ayub \\ BIET, Jhansi \\ Jhansi, UP, India
}

\author{
Vikash Kumar \\ BIET, Jhansi \\ Jhansi, UP, India \\ J P Saini \\ MMEC, Gorakhpur \\ Gorakhpur, UP, India
}

\begin{abstract}
MIT-BIH Database is the standard ECG database which is used universally for ECG analysis purpose. MIT-BIH database for normal sinus rhythm is sampled at $128 \mathrm{~Hz}$ and the data is available at uniform intervals of $7.8125 \mathrm{~ms}$. To use this data for analysis purpose with various techniques like artificial neural networks, correlation techniques etc., it is required to have samples at desired intervals. Hence this paper proposes an image processing method to convert the samples at desired intervals, so that the MIT-BIH database can be used widely and universally.
\end{abstract}

\section{General Terms}

ECG uniform sampling

\section{Keywords}

ECG, Database, Uniform sampling, Image processing.

\section{INTRODUCTION}

The MIT-BIH Normal Sinus Rhythm Database has ECG records in the form of ECG waveform as well as samples as text. The digitization was done using 12 bit analog-to-digital converter (ADC) with sampling frequency $128 \mathrm{~Hz}$ [1] and the samples shown in the database are at intervals of $7.8125 \mathrm{~ms}$ [1]. We require samples at different desired intervals when analysis is done with artificial neural network [2], [3]. The paper proposes a new method to sample the ECG waveforms of the MIT-BIH Normal Sinus Rhythm Database at uniform interval of $8 \mathrm{~ms}$. So that samples at desired intervals can be used by various researchers.

The method we use in this paper to sample the ECG waveforms is based on image processing techniques. Dots per inch (DPI) are used to describe image resolution. For example a 5" $\times 4$ " image digitized at 300 dpi resolution would result in $1500 \times 1200$ dots (pixel) image [4]. For ECG waveform images the image resolution specifies the number of pixels $($ dots $) / \mathrm{mm}$.

Since 1 inch $=25.4 \mathrm{~mm}$,

$$
\text { Number of dots (pixels) } / \mathrm{mm}=\frac{D P I}{25.4}
$$

The modified lead II (MLII) ECG waveforms of the MIT-BIH Normal Sinus Rhythm Database have defined grid intervals (similar to conventional ECG chart paper). On $\mathrm{x}$-axis, i.e. time axis, one grid interval is equal to 0.2 seconds and on y-axis, i.e. voltage axis, one grid interval is equal to $0.5 \mathrm{mV}$. Also the grid interval is of $5 \mathrm{~mm}$. So 1 second corresponds to $25 \mathrm{~mm}$ and $1 \mathrm{mV}$ corresponds to $10 \mathrm{~mm}$. Thus the relationship between pixels and time voltage values can be determined from DPI. For example a $300 \mathrm{dpi}$ corresponds to 11.81 dots (pixels) $/ \mathrm{mm}$. This means that one dot (pixel) corresponds to $3.387 \mathrm{~ms}$ for $\mathrm{x}$-axis i.e. time axis and to $8.47 \mu \mathrm{V}$ for voltage axis and the sampling rate is nearly $295 \mathrm{~Hz}$.

\section{METHODOLOGY}

Uniform samples are extracted from the ECG waveform image. The whole process is summarized in figure 1 by a flowchart.

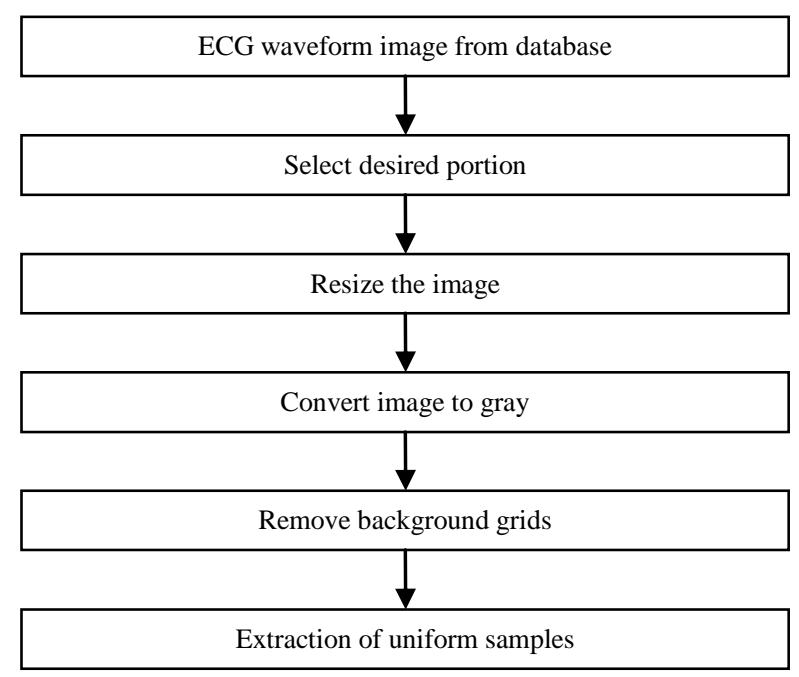

Figure 1 Flow chart describing the uniform sampling method

We describe the method to uniformly sample the ECG waveform with the help ECG waveform of record 16795 from MIT-BIH Normal Sinus Rhythm Database. Figure 2 shows the ECG waveform of record 16795. A portion from the ECG waveform is selected which is to be uniformly sampled, here we have selected the portion from 0 to 2 seconds. The selected desired portion is shown in figure 3 .

\subsection{Resizing of image}

Since we have to achieve a uniform sampling interval of $8 \mathrm{~ms}$ in the data to be extracted from the ECG image, we require that 1 dot (pixel) corresponds to $8 \mathrm{~ms}$ for horizontal axis. So 1 second duration should have 125 dots (pixels). As we have already mentioned that 1 grid interval is of $5 \mathrm{~mm}$ and on time axis, one grid interval is equal to 0.2 seconds and on 


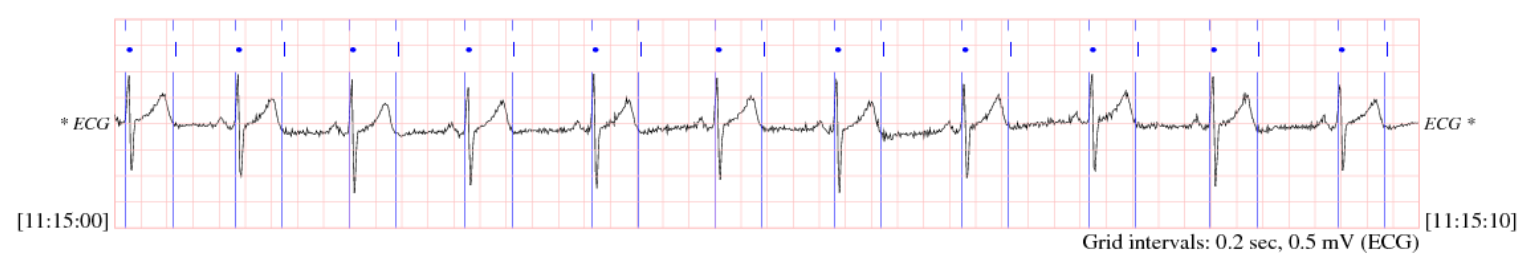

Figure 2 ECG waveform of record 16795 from MIT-BIH Normal Sinus Rhythm Database

voltage axis, one grid interval is equal to $0.5 \mathrm{mV}$. So 1 second corresponds to $25 \mathrm{~mm}$. Therefore in $1 \mathrm{~mm}$ there should be 5 pixels, i.e. number of dots (pixels) $/ \mathrm{mm}=5$. Thus the required resolution is $127 \mathrm{dpi}$.

To get the resolution of 127 dpi we resize the image by 127 percent horizontally as well as vertically, which gives us the image equivalent to resolution of $127 \mathrm{dpi}$ shown in figure 4. We have used Microsoft Windows Paint (version 6.1) to resize the image. Figure 4 shows the resized image of the desired portion.

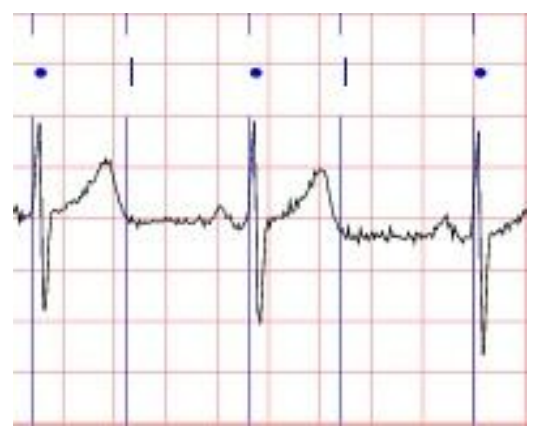

Figure 3 Desired Portion

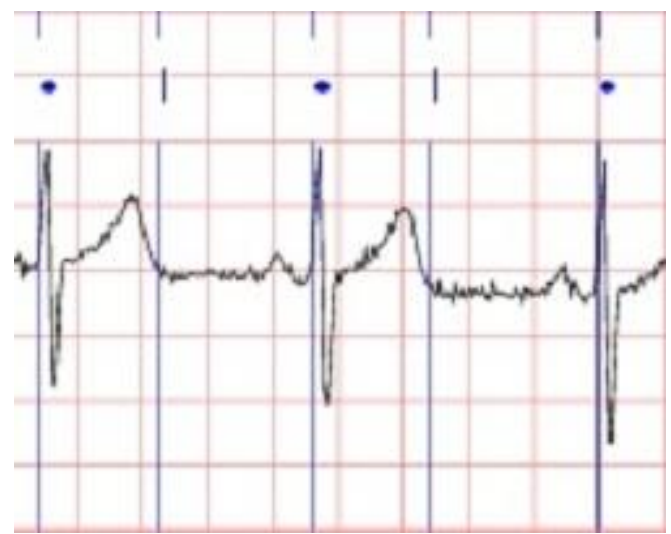

Figure 4 Resized Image

\subsection{Gray conversion of resized image}

The resized image is converted to gray image with the help of MATLAB Simulink [5]. A color space conversion block is used for converting the RGB resized image to intensity image.
Figure 5 shows the gray image obtained after the color conversion.

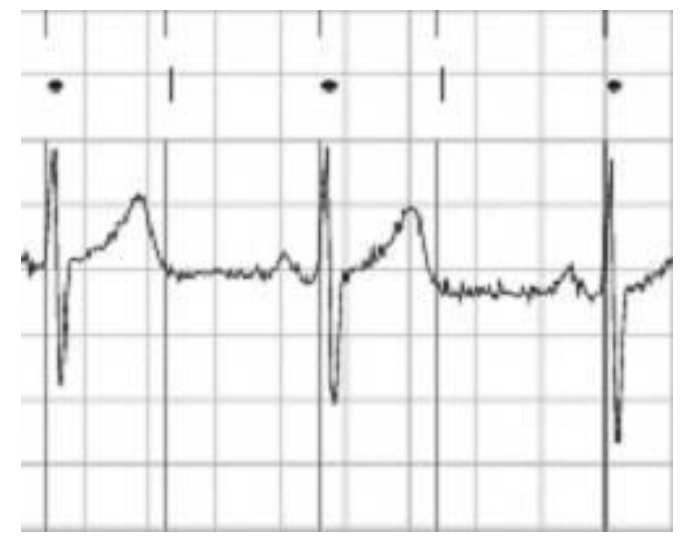

Figure 5 Gray Image

\subsection{Removal of background grids}

We separate background grid from ECG waveform [6], [7]. For removing background grids we have used a thresholder simulink model in MATLAB.

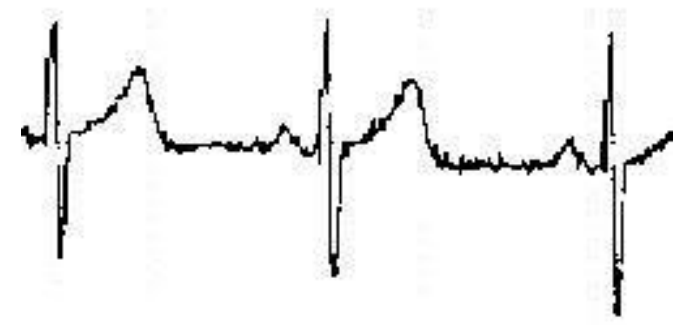

Figure 6 ECG waveform without background

This model based on the pixel values removes all the pixels that have values below an applied threshold. Thus we get an image which has only ECG wave shape with white background as shown in figure 6. If some unwanted dots remain, they should be removed. 


\subsection{Extraction of uniform samples}

The image after background removal is filtered with a contrast-enhancement filter. The ECG waveform is characterized by black pixels. The pixel locations are denoted by $x-y$ Cartesian coordinates. The image shown in figure 6 is transformed from image form to Cartesian coordinates $(\mathrm{x}, \mathrm{y})$ of pixels. Figure 7 shows the pixel plot of ECG waveform in terms of its horizontal and vertical location of its pixels.

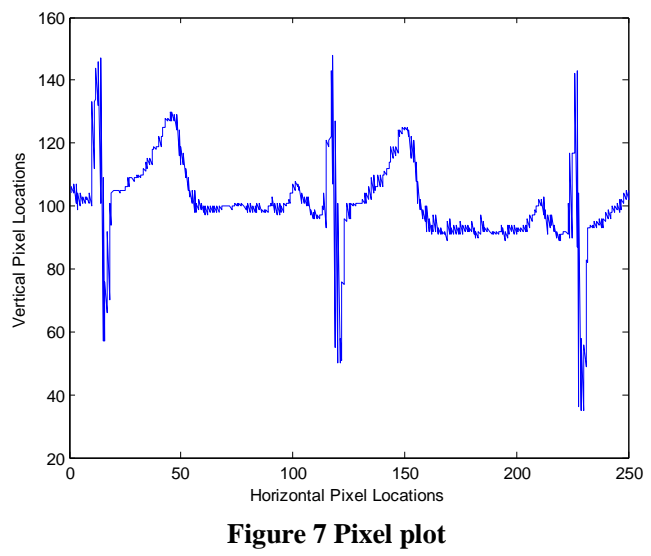

The time and voltage values are obtained by first setting the yaxis reference. Then scaling is done where one dot (pixel) corresponds to $8 \mathrm{~ms}$ for $\mathrm{x}$-axis i.e. time axis and to $0.02 \mathrm{mV}$ for voltage scale. The plot in terms of time and voltage from the ECG image is shown in figure 8 .

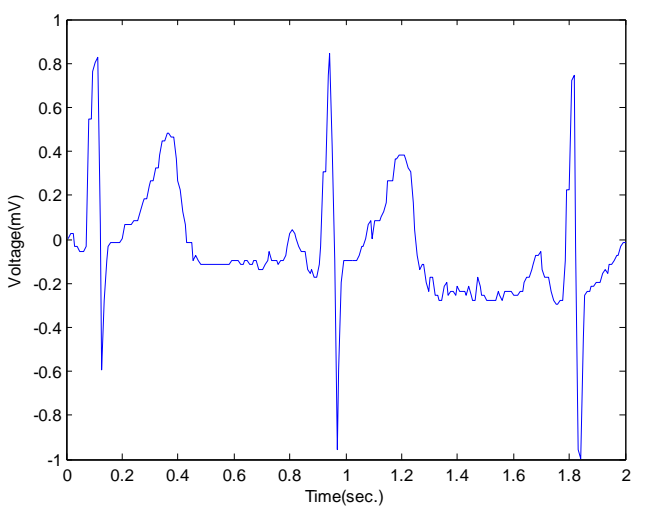

Figure 8 Plot of extracted uniform samples

\section{RESULT}

The samples obtained by our method are at uniform interval of $8 \mathrm{~ms}$. The plot obtained by uniform samples is shown in figure 8 and the plot obtained by samples from database is shown in figure 9. We can see that the plots are similar. A comparison of 30 samples obtained from database and by our method is shown in table 1 . We can see in table 1 that the extracted samples are at uniform interval.

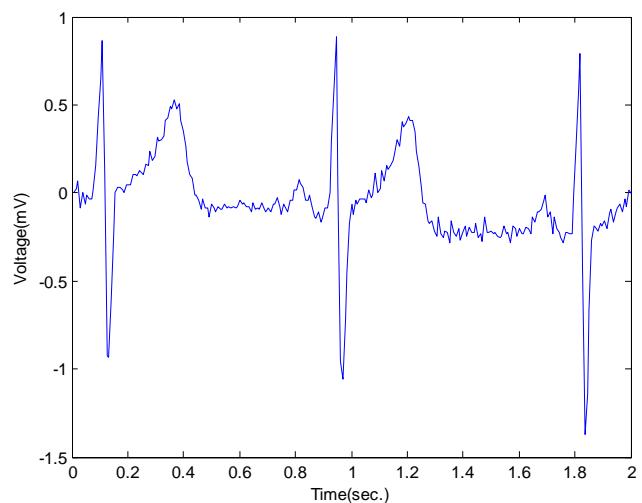

Figure 9 Plot of database samples

Table 1 Samples from database and uniform extracted samples of record 16795

\begin{tabular}{|c|c|c|c|}
\hline \multicolumn{2}{|c|}{$\begin{array}{l}\text { Database samples of } \\
\text { record } 16795\end{array}$} & \multicolumn{2}{|c|}{$\begin{array}{l}\text { Uniform samples of } \\
\text { record } 16795\end{array}$} \\
\hline Time(sec) & Voltage $(\mathrm{mV})$ & Time(sec) & Voltage $(\mathrm{mV})$ \\
\hline 0.008 & 0.005 & 0.008 & 0.005 \\
\hline 0.016 & 0.025 & 0.016 & 0.025 \\
\hline 0.023 & 0.065 & 0.024 & 0.025 \\
\hline 0.031 & -0.085 & 0.032 & -0.035 \\
\hline 0.039 & 0.005 & 0.04 & -0.035 \\
\hline 0.047 & -0.065 & 0.048 & -0.055 \\
\hline 0.055 & -0.015 & 0.056 & -0.055 \\
\hline 0.062 & -0.035 & 0.064 & -0.055 \\
\hline 0.07 & -0.035 & 0.072 & -0.035 \\
\hline 0.078 & 0.015 & 0.08 & 0.545 \\
\hline 0.086 & 0.155 & 0.088 & 0.545 \\
\hline 0.094 & 0.435 & 0.096 & 0.765 \\
\hline 0.102 & 0.655 & 0.104 & 0.805 \\
\hline 0.109 & 0.865 & 0.112 & 0.825 \\
\hline 0.117 & 0.125 & 0.12 & 0.065 \\
\hline 0.125 & -0.925 & 0.128 & -0.595 \\
\hline 0.133 & -0.935 & 0.136 & -0.275 \\
\hline 0.141 & -0.615 & 0.144 & -0.095 \\
\hline 0.148 & -0.235 & 0.152 & -0.035 \\
\hline 0.156 & 0.005 & 0.16 & -0.015 \\
\hline 0.164 & 0.035 & 0.168 & -0.015 \\
\hline 0.172 & 0.035 & 0.176 & -0.015 \\
\hline 0.18 & 0.015 & 0.184 & -0.015 \\
\hline 0.188 & 0.005 & 0.192 & -0.015 \\
\hline 0.195 & 0.045 & 0.2 & 0.005 \\
\hline 0.203 & 0.045 & 0.208 & 0.065 \\
\hline 0.211 & 0.045 & 0.216 & 0.065 \\
\hline 0.219 & 0.105 & 0.224 & 0.065 \\
\hline 0.227 & 0.105 & 0.232 & 0.065 \\
\hline 0.234 & 0.095 & 0.24 & 0.085 \\
\hline
\end{tabular}

\section{LIMITATION OF THIS STUDY}

The samples extracted by our method are at uniform intervals of $8 \mathrm{~ms}$ but due to the blue marker lines available on the ECG waveform image taken from MIT-BIH database, the negative peak values obtained are less in amplitude than the original negative peak values. This will not affect any analysis much because negative peak values are clinically not very much significant. 


\section{CONCLUSION}

The samples obtained from the ECG waveform image by our method are at uniform intervals of $8 \mathrm{~ms}$. Such uniform sampling is useful to use the data for analysis purpose with various techniques like artificial neural networks, correlation techniques etc. Any analysis done by these uniform samples will improve the accuracy. MIT-BIH Arrhythmia database has samples at interval of $(1 / 360) \mathrm{sec}$ whereas MIT-BIH Normal Sinus Rhythm database has samples at interval of (1/128) sec. To use the samples of these databases widely for analysis purpose, it is desired to have samples at a uniform intervals of desired time, which can be obtained by the method proposed in this paper, so that wide database will be available for analysis purpose by any technique.

\section{REFERENCES}

[1] http://www.physionet.org/cgi-bin/atm/ATM, "MIT-BIH Normal Sinus Rhythm Database (nsrdb)".

[2] Shahanaz Ayub, J. P. Saini, 'Fusion Beats extraction from ECG using Neural Network based soft computing Techniques', published in International Journal of Advances in Applied Science Research', Coden (USA),2010,1(2), pp 76-83.
[3] Shahanaz Ayub, J. P. Saini, 'ECG classification and Abnormality Detection using Cascade Forward Neural Network" in International Journal of Engineering, Science \& Technology, 2011,Vol. 3, No. 3, pp. 41-46.

[4] Jalel Chebil, Jamal Al-Nabulsi, Mohammed Al-Maitah, "A Novel Method for Digitizing Standard ECG Papers", Proceedings of the International Conference on Computer and Communication Engineering pp.13081312,2008 .

[5] MATLAB Simulink Help, The MathWorks, Inc., MATLAB 7.12.0 (R2011a).

[6] Sucharita Mitra, M Mitra, "An Automated Data Extraction System From 12 Lead ECG Images", Computer Methods and Programs in Biomedicine, (Elsevier Science publication), vol. 71(1), May(2003), pp 33-38.

[7] Ali S.A. Al-Mejrad, "Bio-Potential Signal Extraction from Multi-Channel Paper Recorded Charts”, American Journal of Applied Sciences, 8(6), pp.520-524. 2011 